\title{
DARK STATES AND DE BROGLIE WAVE OPTICS
}

\author{
H. METCALF \\ Physics Department, State University of New York \\ Stony Brook NY 11794-3800, USA
}

\begin{abstract}
The techniques of laser cooling have now become sufficiently developed that the focus has shifted toward interesting applications such as the quantum domain of atomic motion. This topic is characterized by the failure of the classical description in which atoms move as point particles whose trajectories can be known: instead, atomic motion must be described as the optics of de Broglie waves. For example, when the de Broglie wavelength $\lambda_{\mathbf{d B}}$ exceeds $\lambda_{\text {optical }}$, then a classical description is insufficient (Bose condensation is done in the dark, and the quantum condition becomes $\lambda_{\mathrm{dB}}>$ nearest. neighbor distance). One of the most fascinating topics of quantized atomic motion in a laser field derives from optical dark states that can even occur in the simplest (two-level) atoms, where there are no magnetic sublevels and the polarization is irrelevant. In spite of the simplicity of this two-level atom case however, the more interesting cases occur in multilevel atoms where the internal magnetic states and external quantum states of atomic motion become truly entangled. Schrödinger called such states "the heart of quantum mechanics" because they led to puzzles such as his famous "cat" and the EPR paradox.
\end{abstract}

PACS numbers: $32.80 . \mathrm{Pj}$

\section{Introduction}

In their early days, laser cooling and atom trapping were envisioned as techniques for producing a sample of nearly stationary atoms with negligible Doppler shifts that could be used for precision spectroscopy. More recently, the topic has evolved toward optical control of atomic motion in a broader sense, and has therefore exposed many new and interesting phenomena. The main topic of research in optical control of atomic motion is now no longer the lowest possible steady-state temperatures and the variety of cooling schemes that were of initial interest. Instead, the focus has shifted to the study of elementary processes, especially the quantum mechanical description of the atomic motion.

The uniquely complex character of this subject stems from the internal structure of atoms that can be exploited so beautifully to manipulate their interactions with light. This is accomplished using optical pumping and Raman transitions to optically pump atoms into desired internal states that determine the magnitude and nature of the electromagnetic forces on them. The quantum mechanical description of the atomic motion then means that the Hamiltonian must include 
the kinetic energy of the atomic center-of-mass motion, and that the total wave functions must be composed of both internal and motional contributions.

\section{Laser cooling}

\subsection{Classical view}

From the beginning, theories of laser cooling recognized that dissipation required some irreversible or non-adiabatic behavior. In the extremely simplified but well-studied case of two-level atoms moving in a monochromatic standing wave field, the dissipation is produced by spontaneous emission that provides relaxation between the excited state $|e\rangle$ and the ground state $|g\rangle$ (in a two-level atom, the polarization is irrelevant) [1]. In the spirit of the dressed atom picture [2], the eigenstates of the Hamiltonian are intensity-dependent mixtures of $|g\rangle$ and $|e\rangle$, and atoms moving through an inhomogeneous optical field (e.g., a standing wave) must undergo periodic variation of their internal states to remain in eigenstates of the Hamiltonian. Without fast enough relaxation of the otherwise pure Rabi oscillations, the atoms generally are not in eigenstates.

The required relaxation from spontaneous emission has a rate $\gamma \equiv 1 / \tau$, where $\tau$ is the excited state lifetime, and the spatial inhomogeneity of a standing wave optical field occurs on the scale of $\lambda / 2 \pi$. Thus atomic speeds comparable to $\lambda / 2 \pi \tau \equiv \gamma / k$ result in non-adiabatic response of moving atoms in the field variations they encounter. This can result in energy exchange with the field at each scattering event, of magnitude corresponding approximately to the fraction $v /(\gamma / k)$ of the light shift. For velocities $v \ll \gamma / k$, the internal states can follow the changing field seen by the moving atoms, and the rate of energy exchange is much smaller. For a laser field tuned below an atomic transition, the energy flow is from the atoms into the field, resulting in cooling of the atomic sample. When such laser cooling occurs in pairs of counterpropagating beams, it is called optical molasses, and there are many descriptions of this in terms of the different Doppler shifts seen by the moving atoms [3-5]. The non-adiabatic response, and subsequent energy loss, is then described as a viscous damping force whose nature can be calculated in several ways $[1,6,7]$.

Real atoms have more than two levels, and relaxation to the local eigenstates of such atoms moving in an inhomogeneous optical field can be far more complicated. For example, in a three-dimensional (3D) light field that necessarily has polarization gradients, there can be several relaxation rates among the various Zeeman sublevels of the ground state, and these can be much slower than $\gamma$. Thus there can be significant energy exchange even at velocities much slower than $\gamma / k$, corresponding to laser cooling to much lower temperatures. Such polarization gradient cooling, and other types of related "Sisyphus" mechanisms that routinely cool atomic vapors to the $\mu \mathrm{K}$ regime, have been described in several places [8-12].

\subsection{Quantum view}

Up to now, the motion of atoms has been described in a completely classical way, assuming they had arbitrary position and momentum that could be known simultaneously. The mechanism of energy exchange in laser cooling, as well 
as competition with and limits to that mechanism that determine the ultimate achievable temperatures, depend upon the location of the individual atoms in the spatially varying light field. Such a classical picture of atoms moving as point particles without regard to their overall wave-like character has been of great use, but when atoms are moving sufficiently slowly that their de Broglie wavelength precludes their localization to $\approx \lambda / 2 \pi$, these descriptions fail and a quantum mechanical description is required. Such conditions are routinely achieved in modern laser cooling experiments.

It becomes necessary to consider atomic position and motion as quantum mechanical variables, replete with wave packet spreading and non-commuting operators. A de Broglie wave field occupies allowed states of a region of space that may have a spatially varying potential that derives from the light shift and which defines modes of the field. These may be eigenstates in the optical potentials created by the laser fields. Laser cooling then becomes a process of optically pumping atoms to discrete quantum states of lower kinetic energy [13], dissipating the lost energy by fluorescence into the radiation field. In a standing wave, for example, the light shift produces an array of potential wells and the atoms' quantum states of motion are simply the bound states within these wells as shown in Fig. 1. Laser cooling is then the optical pumping to the lower states as shown [13].

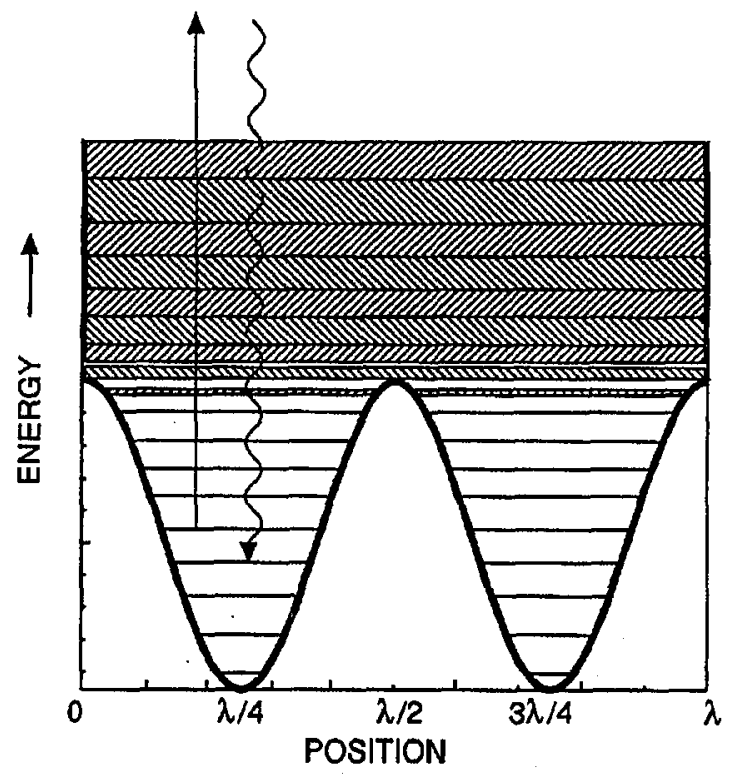

Fig. 1. Energy levels of atoms moving in the periodic potential of the light shift in a standing wave. There are discrete bound states deep in the wells that broaden at higher energy, and become bands separated by forbidden energies above the tops of the wells. Under conditions appropriate to laser cooling, optical pumping among these states favors populating the lowest ones as indicated schematically by the arrows (figure adapted from [13]). 
In analogy with optics, occupation of particular modes of this de Broglie field can result in spatial or temporal interference, and then the entire field of atom interferometry emerges as a subset of this way of thinking. Atoms can only "interfere" if they occupy both the same internal and external states, and thus are truly indistinguishable. "Parts of an atom", i.e., atoms described by a superposition of eigenstates, certainly may have some overlap and can thus partially interfere. Of course, atoms at ordinary thermal velocities are distributed over thousands of quantum states of motion, so laser cooling is intimately involved in these studies. One important difference between this and the optical case arises because unlike photons, atoms are not all bosons. There will be cases where only a single atom can occupy a particular mode of the de Broglie wave field.

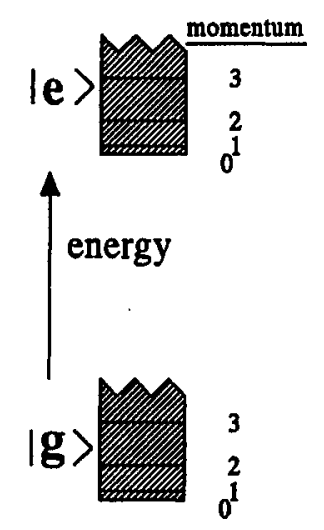

Fig. 2. Kinetic energies accessible to atoms moving freely (no spatially varying light shift) for both ground and excited states, with the integer momentum values marked as lines. All these KE states are doubly degenerate in $\mathrm{ID}$ except for $p=0$.

The quantum description of atomic motion requires that the energy of such motion be included in the Hamiltonian. Such a Hamiltonian will have eigenstates of not only the usual internal energy levels and the atom-laser interaction that connects them, but also of the kinetic energy (KE) operator $P^{2} / 2 M$. These eigenstates will therefore be labelled by quantum numbers of the atomic states as well as the center of mass momentum $p$. For example, an atom in the ground state, $|g ; p\rangle$, has energy $E_{g}+p^{2} / 2 M$ which can take on a range of values. Figure 2 shows the continuum of KE values for both ground and excited states, with the integer momentum values marked as lines. All these $\mathrm{KE}$ states are doubly degenerate in 1D except for $p=0$.

\section{Shedding new light on dark states}

\subsection{Introduction to dark states}

One of the most important requirements for successful experiments in de Broglie wave optics is the preservation of atomic coherence against destruction by spontaneous emission (SE). Recently there have been a few pioneering 
experiments where SE has been carefully avoided by detuning the light frequency $\omega_{\ell}$ so very far from atomic resonance $\omega_{\mathrm{a}}$ that the rate of SE becomes negligibly small: only a few atoms are lost from the experiment because of SE. Thus atoms are kept "in the dark".

Using large values of $\delta \equiv \omega_{\ell}-\omega_{\mathrm{a}}$ is not the only way to inhibit SE; a far more interesting way involves inherently "dark states", atomic states that cannot be excited by the light field. Some atomic states are trivially dark, that is, they cannot be excited because the light has the wrong frequency or polarization. The more interesting cases are superposition states created by coherent optical Raman coupling $[14,15]$. A very special case are those superpositions whose excitable component vanishes exactly when their external (de Broglie wave) states are characterized by a particular momentum. Such velocity selective coherent population trapping (VSCPT) has been a subject of considerable interest since its first demonstration in 1988 [16-18]. VSCPT enables arbitrarily narrow momentum distributions and hence arbitrarily large delocalization for atoms in the dark states.

\subsection{The two-level atom case}

To see how the quantization of atomic motion allows the existence of such a velocity selective dark state, we consider the states of a two-level atom with single internal ground and excited levels, $|g ; p\rangle$ and $|e ; p\rangle$. Two ground eigenstates $|g ; p\rangle$ and $\left|g ; p^{\prime}\right\rangle$ are generally not coupled to one another by an optical field except in certain cases. For example, in oppositely propagating light beams (1D) there can be absorption-stimulated emission cycles that connect $|g ; p\rangle$ to itself or to $|g ; p \pm 2\rangle$, depending on whether the stimulated emission is induced by the beam that excited the atom or by the other one (momentum is measured in units of $\hbar k$ ).

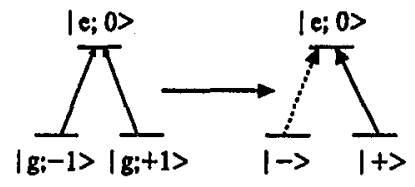

Fig. 3. Schematic diagram of transformation of eigenfunctions from the internal atomic states $|g ; p\rangle$ coupled by Raman transitions to the coupled basis $| \pm\rangle$.

In the first case, the states of the atom and field are left unchanged, but the interaction shifts the internal atomic energy levels, thereby producing the light shift. In the second case, the initial and final $\mathrm{KE}$ of the atom differ by $\pm 4(p \pm 1) / 2 M$ (the energy of the light field is unchanged by the interaction), so energy conservation requires $p=\mp 1$. Thus energy conservation corresponds to Raman resonance between the distinct states $|g ;-1\rangle$ and $|g ;+1\rangle$, and is therefore velocity selective. The coupling of these two degenerate stationary states by the light field results in the new ground eigenstates of the (optical $+\mathrm{KE}$ ) Hamiltonian given by (see Fig. 3)

$$
| \pm\rangle \equiv \frac{|g ;-1\rangle \pm|g ;+1\rangle}{\sqrt{2}}
$$


Excitation of the states $| \pm\rangle$ given in Eq. (1) to $|e ; 0\rangle$ is proportional to the square of the radial matrix element

$$
|\langle e ; 0|r| \pm\rangle|^{2}=\frac{|\langle e ; 0|r| g ;-1\rangle \pm\langle e ; 0|r| g ;+1\rangle|^{2}}{2}
$$

and this vanishes for $|-\rangle$ because the two terms on the right side of Eq. (2) are equal since $r$ does not operate on the external momentum of the atom (dashed line of Fig. 3). Excitation of $| \pm\rangle$ to $|e ; \pm 2\rangle$ is off resonance because its energy is higher by $4 \hbar \omega_{\mathrm{r}} \equiv 4 \hbar^{2} k^{2} / 2 M$ so that the required frequency is higher than to $|e ; 0\rangle$. The detuning is thus $8 \omega_{\mathrm{r}} / \gamma \equiv 8 \epsilon$ halfwidths, and for $\epsilon \approx 0.5-1$, this is large enough so that the excitation rate is small, making $|-\rangle$ quite dark. Excitation to any state of $p^{\prime} \neq 0, \pm 2$ violates conservation of momentum and is forbidden. Atoms are therefore optically pumped into the dark state $|-\rangle$ where they stay trapped, and since their momentum components are fixed, the result is VSCPT [17].

A useful view of this dark state arises by considering that its components $|g ; \pm 1\rangle$ have well defined momentum, and are therefore completely delocalized. Such oppositely travelling waves of the same frequency form a standing de Broglie wave. The fixed spatial phase of this wave relative to the optical standing wave results in the vanishing of the spatial integral of the dipole transition matrix element so that the state cannot be excited.

This view can also help to understand the consequences of $p$ not exactly equal to \pm 1 . In this case, the two components of the standing wave do not have exactly the same energy, and so their relative phase evolves at the rate of their energy difference. The de Broglie wave "walks" at a corresponding velocity, and has soon shifted its phase relative to the optical standing wave by $\pi / 4$. Thus its transition matrix element no longer vanishes, and the "evolved" state is readily excited by the standing wave laser field.

\subsection{Bragg reflection}

A different view of VSCPT emerges by considering more carefully the motion of such dark state atoms in the spatially periodic field of oppositely propagating light beams [19]. As Fig. 4 shows, dark state atoms travelling with longitudinal

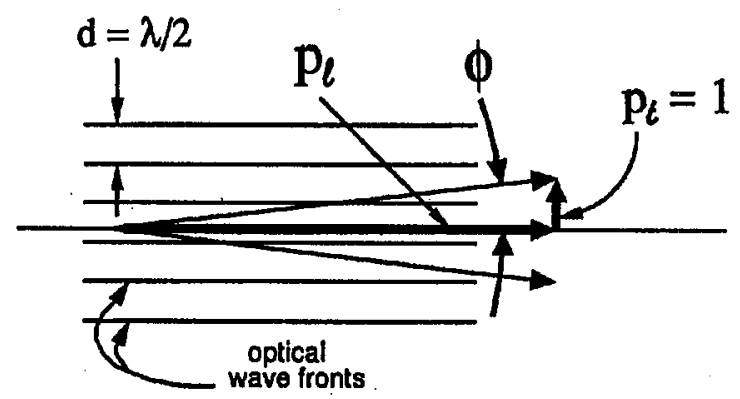

Fig. 4. The trajectory of an atom in the dark state makes an angle $\phi$ with the wave front planes. Its transverse component is one recoil. 
momentum $p_{\ell}$ make an angle $\phi$ with the optical wave fronts, and their de Broglie wavelength is

$$
\lambda_{\mathrm{dB}}=\frac{h}{\sqrt{p_{\ell}^{2}+1}}=\frac{2 \pi}{k} \sin \phi
$$

since $\sin \phi=1 / \sqrt{p_{\ell}^{2}+1}$ (momentum is in units of $\hbar k$ ). Thus

$$
\lambda_{\mathrm{dB}}=2 d \sin \phi
$$

where $d \equiv \lambda / 2$ is the spatial periodicity of the light field. Equation (4) is exactly the equation for Bragg diffraction, but its interpretation in this context is indeed most astounding [19]. Here the de Broglie "matter" wave is Bragg diffracted by the spatially periodic optical field: matter and field have been interchanged from the usual case of Bragg diffraction of an electromagnetic field by crystalline planes of atoms!

In retrospect, if we view ordinary Bragg diffraction as arising from multi-center scattering of radiation by atoms at each lattice site, then propagation of the diffracted wave can occur only in the preferred direction defined by Eq. (4). Such waves are the only ones not diffusively scattered by the lattice. The equivalent view of atoms in dark states is simply that the de Broglie wave fields propagate without scattering (i.e., no SE) in the light field only when the atoms are indeed in dark states [19]. Such an effect has recently been observed in metastable $\mathrm{Ar}$ atoms [20].

Such Bragg reflection has a rather simple intuitive explanation. In the absence of $\mathrm{SE}$, atoms cannot exchange energy with the light field since its energy is unchanged by stimulated emission processes (the frequency is always $\omega_{\ell}$ ). Therefore atoms entering the light field with momentum $p_{t}$ can leave with only $\pm p_{t}$. Their paths are thus either unaltered or Bragg reflected, and either process preserves coherence. Beam splitting simply leaves atoms in a superposition of degenerate states with $+p_{t}$ and $-p_{t}$.

\subsection{High velocity dark states}

The discussion above has focussed on the case of dark states composed of superpositions that arise from a two-photon coupling between two states whose momenta differ by \pm 2 . It is also possible to construct analogous dark states with $2 n$-photon coupling between two states whose momenta differ by $\pm 2 n$. Of course, their energies are larger, but as long as the components have equal energies, that is, their opposite momenta have equal magnitude, the states are stationary and remain dark. For $n=2$, the two momentum states having $p= \pm 2$ have momenta that differ by 4 , and so a four-photon Raman transition is required to conserve momentum, corresponding to a higher order process in VSCPT.

Experimentally we note that population always accumulates in the state $|-\rangle$ because it is dark, producing peaks in the momentum distribution at $p= \pm 1$. But there is also population accumulated in the analogous superposition states coupled by four photons that produce peaks in the momentum distribution at $p= \pm 2$, resulting in four peaks as shown in Fig. 5. Both of these long-lived states are populated by a random walk in momentum space, and each of them has a 


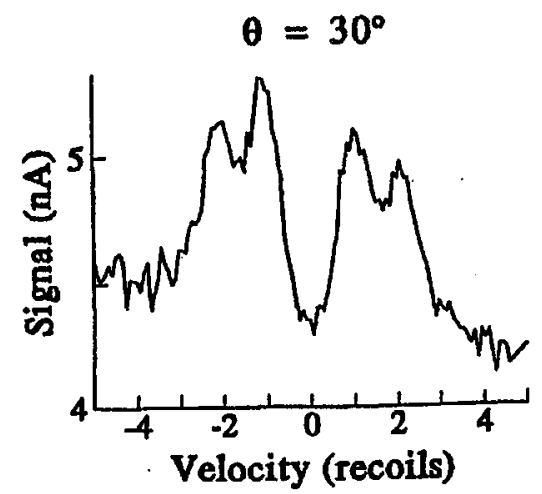

Fig. 5. Measured $\mathrm{He}^{*}$ velocity distributions after $20 \mu$ s interaction time for $I=$ $1.6 \mathrm{~mW} / \mathrm{cm}^{2}$ and zero detuning with counterpropagating beams linearly polarized at $30^{\circ}$. The four-peaked structure is quite evident.

long enough lifetime to be readily observable in an experiment with appropriate interaction time. Thus we see four very narrow $(F W H M \leq 1)$ peaks in the measured momentum distribution of Fig. 5 [21]. From the point of view of Bragg reflection as described above in Sec. 3.3 above, this simply corresponds to the next higher order, found by replacing the right hand side of Eq. (4) by $2 n d \sin \phi$ with $n=2$. This is the only example we know of using higher order non-linear optical effects to produce dark states and laser cooling.

\section{Real (multilevel) atoms \\ 4.1. The $J=1 \Leftrightarrow 1$ transition}

Real atoms have multiple internal levels that include the magnetic, hfs, and other sublevels, and thus the strength of their optical interactions depends on the light polarization. A particularly beautiful example of dark states appears in the $J=1 \Leftrightarrow 1$ transition, where the optical selection rules associated with $M_{J}$ produce an analog of the hypothetical state $|-\rangle$ that is perfectly dark [16-18].

When a single circularly polarized light beam drives a $J=1 \Leftrightarrow 1$ transition with $\Delta M_{J}=+1$, the state $M_{J}=+1$ is dark, and similarly for $\Delta M_{J}=-1$ and $M_{J}=-1$ with the opposite circular polarization. (Choosing the $z$-axis parallel to the beam's $k$ vector allows only $\Delta M= \pm 1$ transitions because the light has no electric field component parallel to $k$.) Because the transition $M_{J}=0 \rightarrow 0$ is forbidden by the selection rules, the $M_{J}=0$ level is then emptied by optical pumping, and the only populated ground states are $M_{J}= \pm 1$ and the excited state $M_{J}=0$, forming " $\Lambda$ " system of levels.

The velocity-dependent dark state can be visualized by considering that a single beam travelling in the $+z$ direction excites the ground state $\left|M_{J}=-1 ; p-1\right\rangle$ only to a single excited state $\left|M_{J}=0 ; p\right\rangle$ as shown in Fig. 6 (the excited state $\left|M_{J}=0 ; p-2\right\rangle$ is not coupled by a beam travelling in the $+z$ direction). A beam of opposite circular polarization travelling in the opposite direction excites $\left|M_{J}=+1 ; p^{\prime}+1\right\rangle$ only to a single excited state $\left|M_{J}=0 ; p^{\prime}\right\rangle$. The two excited 


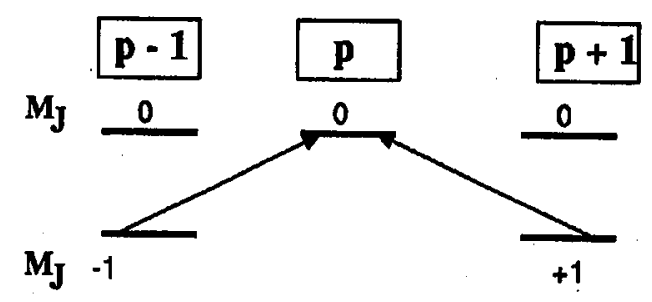

Fig. 6. The coupling between momentum states connected by oppositely circularly polarized light beams travelling in opposite directions. The internal atomic states are indicated by their $\dot{M}_{J}$ values, and in this $J=1 \Leftrightarrow 1$ transition, they are entangled with the momentum states. The $\Lambda$ system consists of the set of three levels coupled by the two arrows.

states are the same only for $p=p^{\prime}=0$, and the subsequent mixing of the ground states forms two new superposition states, as in Sec. 3.2. These states are given by

$$
| \pm\rangle=\frac{|+1,+1\rangle \pm|-1,-1\rangle}{\sqrt{2}},
$$

where the quantum numbers in the kets are $\left|M_{J}, p\right\rangle$.

\subsection{Entanglement}

One of the most interesting aspects of dark state physics arises from the entanglement of motional and internal states that can be produced. The states represented in Eq. (5) are called entangled states because they satisfy the very special criterion that there exists no basis set in which the states could be written as a product of sums. This leads to the opportunity for fundamental studies at the heart of quantum mechanics, such as quantum communication, computing, cryptography, and teleportation.

Although there have been many recent studies of these topics using photons as the primary quantum objects, the use of atoms has many advantages. Atomic dark states have special advantages. First, the number state can be controlled with minimal doubt, unlike optical fields whose Fock states always have significant uncertainty. Perhaps more important, the number of Hilbert spaces, as well as their dimensionality, can each be large than two.

As an illustration of how these entangled states could enable quantum computing, we consider the elementary functional unit of a quantum computer, the controlled NOT gate (CN). A two-qubit CN gate, which can be combined with rotations to enable any computational operation, can be realized directly using the states $\left|M_{J}, p\right\rangle$. By simply applýing a focused optical Raman $\pi$-pulse to one of the two separated VSCPT beams, say $|+1,+1\rangle$, the following CN truth table results:

\begin{tabular}{|lll|}
\hline$A=|-1,-1\rangle$ & $\rightarrow$ & $A^{\prime}=|-1,-1\rangle$ \\
\hline$B=|+1,-1\rangle$ & $\rightarrow$ & $B^{\prime}=|+1,-1\rangle$ \\
\hline$C=|+1,+1\rangle$ & $\rightarrow$ & $C^{\prime}=|-1,+1\rangle$ \\
\hline$D=|-1,+1\rangle$ & $\rightarrow$ & $D^{\prime}=|+1,+1\rangle$ \\
\hline
\end{tabular}


Clearly the value of $p$ controls the inversion of $M_{J}$. This neutral atomic beam version is complementary to a quantum $\mathrm{CN}$ gate realized with trapped ions, while retaining the relatively high isolation from environmental decoherence (the momentum states are naturally very robust, and the internal states are composed entirely of ground levels).

Note that the Raman $\pi$-pulse converts the perfectly entangled state $(A+C) / \sqrt{2}$ into the product state $\left(A^{\prime}+C^{\prime}\right) / \sqrt{2}(=(A+D) / \sqrt{2})$, and vice versa. That is, the state $|-\rangle$ given in Eq. (5) is converted into

$$
\frac{\left|M_{J}=-1\right\rangle \otimes(|p=+1\rangle+|p=-1\rangle)}{\sqrt{2}} \text {. }
$$

Because the states $A$ and $C$ are orthogonal, overlapping them would produce interference fringes of visibility equal to zero because their superposition is a perfectly entangled state [22], whereas overlapping states $A^{\prime}$ with $C^{\prime \prime}$ would produce fringes of visibility equal to unity because their sum can be written as a simple product as in Eq. (6).

\section{Conclusions and summary}

Advances in laser cooling have imposed a new view of atomic motion that constitutes de Broglie wave optics. Optical pumping of atoms among the momentum states accessible to these de Broglie waves shares many commui features with optical pumping among internal atomic states. Especially interesting features arise when entangled superpositions of momentum and internal states result in dark states that can be readily observed because of their long lives.

This paper is not a report of work done solely by the author, but rather by his students and associates. The work described here was done principally by Marya Doery, Mark Widmer, Mary-Jo Bellanca, Wally Buell, Edgar Vredenbregt, and Tom Bergeman. The research was supported by the NSF and the ONR.

\section{References}

[1] J. Gordon, A. Ashkin, Phys. Rev. A 21, 1606 (1980).

[2] C. Cohen-Tannoudji, S. Reynaud, J. Phys. B 10, 345 (1977).

[3] D. Wineland, W. Itano, Phys. Rev. A 20, 1521 (1979).

[4] J. Dalibard, W. Phillips, Bull. Am. Phys. Soc. 30, 748 (1985).

[5] S. Chu, L. Hollberg, J. Bjorkholm, A. Cable, A. Ashkin, Phys. Rev. Lett. 55, 48 (1985).

[6] H. Metcalf, P. van der Straten, Phys. Rep. 244, 203 (1994).

[7] M. Doery, E. Vredenbregt, T. Bergeman, Phys. Rev. A 51, 4881 (1995).

[8] P. Lett, R. Watts, C. Westbrook, W. Phillips, P. Gould, H. Metcalf, Phys. Rev. Lett. 61, 169 (1988).

[9] J. Dalibard, C. Cohen-Tannoudji, J. Opt. Soc. Am. B 6, 2023 (1989).

[10] P. Ungar, D. Weiss, E. Riis, S. Chu, J. Opt. Soc. Am. B 6, 2058 (1989).

[11] C. Cohen-Tannoudji, W. Phillips, Phys. Today 43, 33 (1990).

[12] G. Nienhuis, P. van der Straten, S.-Q. Shang, Phys. Rev. A 44, 462 (1991). 
[13] T. Bergeman, Phys. Rev. A 48, R3425 (1993).

[14] E. Arimondo, G. Orriols, Lett. Nuovo Cim. 17, 333 (1976).

[15] H. Gray, R. Whitley, C. Stroud, Opt. Lett. 3, 218 (1978).

[16] A. Aspect, E. Arimondo, R. Kaiser, N. Vansteenkiste, C. Cohen-Tannoudji, Phys. Rev. Lett. 61, 826 (1988).

[17] A. Aspect, E. Arimondo, R. Kaiser, N. Vansteenkiste, C. Cohen-Tannoudji, J. Opt. Soc. Am. B 6, 2112 (1989).

[18] F. Bardou, J. Bouchard, O. Emile, A. Aspect, C. Cohen-Tannoudji, Phys, Rev. Lett. 72, 203 (1994).

[19] H. Batelaan, S. Bernet, M. Oberthaler, E. Rasel, J. Schmiedmayer, A. Zeilinger, in: Advances in Atomic, Molecular, and Optical Physics: Supplement Atom Interferometry, Ed. P.R. Berman, Academic Press, to be published.

[20] S. Bernet, M. Oberthaler, R. Abfalterer, J. Schmiedmayer, A. Zeilinger, Phys. Rev. Lett. 77, 5160 (1996).

[21] M. Widmer, M. Doery, M.-J. Bellanca, W. Buell, T. Bergeman, H. Metcalf, Phys. Rev. A 53, 946 (1996).

[22] M. Scully, B.-G. Englert, H. Walther, Nature 351, 111 (1991). 